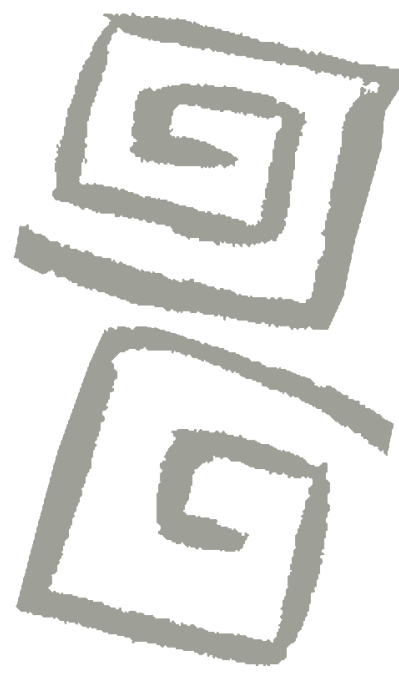

\title{
El impacto de la reforma Cabanis en la formación histórica de la universidad argentina y en la educación superior en salud
}

\author{
The impact of the Cabanis reform on the historical \\ formation of the Argentine university and on higher \\ education in health
}

Naomar Almeida-Filho', Mariano Di Pasquale²

${ }^{1}$ Médico. Doctor en Epidemiología, Doctor of Science (honoris causa) Profesor titular (jubilado), Instituto de Saúde Coletiva, Universidade Federal da Bahia, Brasil. Investigador Senior (nivel I-A), Consejo Nacional de Desarrollo Científico y Tecnológico, Brasil. $\triangle$ iD

${ }^{2}$ Docteur en Histoire et civilisations. Profesor

adjunto, Instituto de Estudios Históricos, Universidad Nacional de Tres de Febrero. Investigador asistente, Consejo Nacional de Investigaciones Científicas y Técnicas, Buenos Aires, Argentina. $\bowtie$ i
RESUMEN En este ensayo, proponemos evaluar el impacto de la reforma Cabanis en la configuración del modelo de formación en salud que se hizo hegemónico en Argentina. Primero, analizamos la reestructuración del sistema educacional francés desencadenada por la revolución de 1789, principalmente en sus dimensiones político-institucionales. Segundo, discutimos brevemente el proceso de reconstrucción social, ideológica e institucional del sistema de salud en la Francia postrevolucionaria. Tercero, introducimos la reforma Cabanis, propuesta de enseñanza médica basada en profesionalismo, disciplinariedad y especialización que resultó en un sistema de enseñanza superior sin universidades, e indagamos en torno a sus principales aspectos curriculares y pedagógicos. Finalmente evaluamos la secuencia de eventos y procesos que, durante todo el siglo XIX, con fuerte gravitación cabanisiana a través de la Idéologie, conformaron los rasgos principales tanto de la enseñanza de la salud y de la medicina como del modelo de educación superior basado en facultades que actualmente predomina en la República Argentina.

PALABRAS CLAVES Educación en Salud; Historia de la Medicina; Revolución Francesa; Argentina.

\begin{abstract}
In this essay, we seek to evaluate the impact of the Cabanis reform on the configuration of the health education model that became hegemonic in Argentina. First, we analyze the restructuring of the French educational system triggered by the revolution of 1789 , primarily in its political and institutional dimensions. Second, we briefly discuss the process of the social, ideological and institutional reconstruction of the health system in post-revolutionary France. Third, we introduce the Cabanis reform, a proposal for medical education based on professionalism, disciplinarity and specialization that resulted in a higher education system without universities, looking into its main curricular and pedagogical aspects. Finally, we evaluate the sequence of events and processes that, throughout the 19th century, with strong Cabanisian influence through the Idéologie, shaped both the principal features of teaching health and medicine and the higher education model based on faculties currently predominant in the Argentine Republic.
\end{abstract} KEY WORDS Health education; History of Medicine; French Revolution; Argentina. 


\section{INTRODUCCIÓN}

Entre los siglos XVII y XVIII, se consolidó en todo el mundo occidental un modelo de educación superior vocacional, cuya misión era formar cuadros capaces de operar la gestión del Estado absolutista moderno ${ }^{(1)}$. Este período constituye, en la historia de las ideas, una época en que la institución llamada universidad, fuertemente impregnada por el poderío de la religión, se hacía conservadora y resistía a la ciencia; a pesar de ser, paradójicamente, un momento de explosión del conocimiento científico en el mundo. En ese escenario de profundos cambios económicos y políticos, con la emergencia de un nuevo modo de producción, el ascenso de clases sociales antes subalternas y la configuración de nuevas formas de gobierno, la ya secular institución de conocimiento pasó a enfrentar una seria crisis de identidad y finalidad social. Cuando la Asamblea Nacional suprimió las universidades francesas, en un momento de gran turbulencia política y rápido cambio social, la Revolución Francesa produjo una solución política radical para esa crisis. En contraste, la solución germánica y posteriormente anglosajona para superar la crisis implicaron un retorno activo a los principios originales de la universidad como concepto político estratégico, con la creación del modelo de universidad de investigación a través de la reforma Humboldt, a partir de $1810^{(2)}$.

La reestructuración de la educación en la Francia posrevolucionaria, delineada en los rapports de Mirabeau y Condorcet, fue profundamente influenciada por la obra filosófica y por el trabajo político de Pierre-JeanGeorges Cabanis [1757-1808]. Modelado en la Idéologie e implantado por la reforma bonapartista, el sistema francés de enseñanza pública aparecía como paradigma para otros pueblos sometidos a la poderosa influencia cultural francesa, durante todo el siglo XIX. Con la ruptura del modelo de universidad formadora de cuadros para el Estado-nación aristocrático, realizada por la Revolución Francesa y consolidada en la reforma educativa de Bonaparte, un proceso de ajuste de los respectivos modelos de educación superior se inició en todo el espacio cultural de influencia intelectual francesa, principalmente en la Europa meridional y países recién salidos de la condición de colonia.

En América Latina, Gregorio Weinberg distingue el modelo hispano del modelo lusitano de política educativa colonial| ${ }^{(3)}$. Para él, la colonización española promovía la apertura de universidades como una solución para el control político y religioso de un enorme territorio, subdividido en muchas provincias, distantes y diversas entre sí. Esa política fue bien distinta, pero no menos efectiva que la adoptada por la colonización portuguesa que, considerando la unidad geopolítica de su colonia principal, Brasil, logró imponer un monopolio ideológico e intelectual rígido, al prohibir a la prensa y la educación superior durante más de tres siglos ${ }^{(3)}$. En la medida en que son representativos de diferentes versiones del modelo mediterráneo de educación superior, adaptados a distintos contextos postcoloniales, dos países merecen atención especial: Brasil y Argentina.

Mientras la situación de Brasil es analizada en otro texto ${ }^{(4)}$, en este ensayo proponemos evaluar las raíces históricas del modelo de formación en salud que se hizo hegemónico en Argentina. Para ello, en primer lugar, analizamos la reestructuración del sistema educacional francés desencadenada por la revolución de 1789, principalmente en sus dimensiones político-institucionales. En segundo lugar, discutimos brevemente el proceso de reconstrucción social, ideológica e institucional del sistema de salud en la Francia postrevolucionaria. A continuación, introducimos los principales elementos estructurales de la reforma Cabanis, propuesta de enseñanza médica basada en profesionalismo, disciplinariedad y especialización que resultó en un sistema de enseñanza superior sin universidades, e indagamos en torno a sus principales aspectos curriculares y pedagógicos. Finalmente, evaluamos la secuencia de eventos y procesos que, durante todo el siglo XIX, con fuerte gravitación cabanisiana a través de la Idéologie, conformaron tanto los rasgos principales de la enseñanza de la 
salud y de la medicina como así también el modelo de educación superior basado en facultades que actualmente predomina en la República Argentina.

\section{ANTECEDENTES}

Antes de la Revolución Francesa de 1789, la monarquía era asumida como legado divino, la institución escolar era tutelada por la Iglesia católica romana y la religión se declaraba propósito último de la educación ${ }^{(5)}$. En sus primeros actos deliberativos, al retirar del clero la prerrogativa de gestión de la enseñanza y al extinguir las universidades, la Asamblea Nacional tomó una decisión radical de política educativa. En vez de instituciones de enseñanza al servicio de una aristocracia diletante y alienada, la naciente república necesitaría más de una red eficiente de órganos públicos para la formación política, técnica y profesional de ciudadanos libres. En este contexto, la educación superior, basada en instituciones de gran porte y centralizadas, formadas por la elite intelectual y directamente regidas por el poder real, era criticada por su anacrónica tradición escolástica y su indiferencia (e incluso reacción) al avance de las ciencias modernas ${ }^{(6)}$.

\section{La reforma de la educación y la Idéologie}

En 1790, la Convención Nacional nombró un Comité d'instruction publique para coordinar la reordenación de la educación en Francia, teniendo como prioridad política reducir la influencia religiosa en la formación de los ciudadanos. Como resultado inicial de ese trabajo, la Constitución francesa, aprobada en 1791, incluyó la educación como derecho de todos, garantizada mediante "una instrucción pública común a todos los ciudadanos $^{\prime \prime(7)}$. En 1792, el Marqués de Condorcet [1743-1794] sometió a la Asamblea Nacional un plan de reorganización del sistema de educación, bajo los principios políticos de la Revolución Francesa( ${ }^{(8)}$. Como apéndice, incluía una propuesta presupuestaria y un estudio de viabilidad, además de un borrador de decreto legislativo que, de forma minuciosa, establecía desde una base curricular común hasta programas de becas de permanencia y movilidad estudiantil. El grado de detalle confirma la construcción colectiva del proyecto. A pesar de todo eso, aun siendo presentada en nombre del Comité, esa propuesta fue rechazada.

Con el agotamiento de la turbulenta política del Terror, en 1794, al alcanzar mayor estabilidad institucional, el gobierno revolucionario pudo finalmente priorizar la reforma educativa. François Daunou [1761-1840] redactó una propuesta de ley orgánica de la educación pública, sometida a la Convención en octubre de 1795 y luego aprobada. Para todo el país, se propuso un modelo centralizado de educación, parcialmente pública, con apertura de escuelas centrales de enseñanza media, que incluían biblioteca pública, jardín botánico y museo de historia natural. A pesar de ese esfuerzo de reorganización, la calidad de la educación pública en la Francia posrevolucionaria seguía siendo precaria y la situación de la enseñanza, cada vez más preocupante, evidenciaba el fracaso de una política educativa focal y centralizadora( ${ }^{(7)}$.

En 1796, Cabanis y su amigo Destutt de Tracy [1754-1836] promovieron una agrupación de jóvenes pensadores que se conoce como Les idéologues ${ }^{(9,10)}$. Adelantándose a la noción contemporánea de "democracia cognitiva", los ideólogos defendían que solo habría progreso social con igualdad intelectual para todos los seres humanos, mediante el amplio acceso al conocimiento a través de una educación libertadora. Consideraban la instrucción pública como crucial en una república fundada en los principios de libertad e igualdad individuales, en que todos los ciudadanos tuvieran una comprensión adecuada de sus derechos y deberes, así como plena capacidad para participar en procesos deliberativos, de evaluación de dirigentes y del juicio de la representatividad política. Una nueva política, a su vez, requeriría una ciencia fundada en una epistemología 
revolucionaria, verdadera "ciencia de los métodos" que implicaría una lógica de los modos de pensamiento y una fisiología de los sistemas psíquicos. Esta nuova scienza comienza a delinearse en el ambicioso compendio de síntesis entre filosofía, gramática y lógica de Destutt de Tracy ${ }^{(11)}$ y en el desarrollo de una ciencia moral basada en la medicina. Cabanis contribuiría decisivamente a profundizar esa perspectiva sensualista y naturalista, con un abordaje original de la cuestión cartesiana de la relación cuerpo-mente en su Science de $I^{\prime}$ homme ${ }^{(12,13)}$. Al proponer abordar las complejas interacciones sociales con una mirada biomédica, Cabanis no pretendía reducir lo físico a la determinación orgánica de la vida, ni lo moral al aparato jurídico-legal que lo conforma en una normatividad vital. En esta perspectiva, lejos de ser comprendido básicamente por el estudio físico del hombre y por el estudio histórico de sus normas y leyes, el "punto de vista moral" necesita ser entendido de modo ampliado, ciertamente lejos del sentido común de moralismo y moralidad ${ }^{(14)}$.

La Idéologie sostiene que los seres humanos son orientados hacia la perfectibilité -término acuñado por Jean-Jacques Rousseau [1712-1778] para designar una tendencia a la búsqueda de la perfección, innata entre los humanos ${ }^{(14)}$ - al mismo tiempo natural y cultural, en el sentido de constante perfeccionamiento como individuo y como especie (en el léxico de la época, utiliza el término race), en dos aspectos: físico y moral. El primero implica evolución de las capacidades individuales heredadas, desde una perspectiva claramente lamarckiana. Según Staum ${ }^{(15)}$ Cabanis adelantó algunas ideas y, al mismo tiempo, fue influenciado por su contemporáneo Jean-Baptiste de Lamarck [1744-1829], famoso por la teoría de evolución de las especies vía la transmisión genética de caracteres adquiridos, divulgada en 1809 .

La herencia de características adquiridas significaba que los seres humanos serían capaces de transformar su propia naturaleza para que, a lo largo de varias generaciones, "no existan los mismos hombres ni la misma raza"; los progresos sociales, morales y políticos introducirían una gran distancia entre los diferentes hombres con "disposiciones primitivas iguales"(12). El segundo aspecto se refiere a la determinación social del perfeccionamiento humano, viabilizado por políticas educativas universalistas, por ello su interés en reformas de la educación pública ${ }^{(14)}$.

\section{Vicq-d'Azyr, Fourcroy y la reforma de la medicina}

En ese momento de emergencia del capitalismo industrial, el escenario francés de asistencia a la salud revelaba una situación de enorme desigualdad y segregación social $^{(6,16,17)}$. La población pobre tenía acceso a cuidados de salud prestados por barberoscirujanos y boticarios, que circulaban por las ferias del interior del país haciendo pequeñas cirugías, reduciendo fracturas, extrayendo dientes y dispensando medicinas, en general, fitoterapéuticos. Bajo la gestión de las órdenes religiosas, los hospitales eran lugares de recepción de los moribundos, aunque también funcionaron como albergues para los viajeros y vagabundos, o como manicomios. En muchos casos, sirvieron como prisiones políticas y pocos se ocuparon del cuidado y de la curación ${ }^{(17,18)}$. En esa coyuntura, la nobleza era atendida por médicos clínicos (llamados físicos) o cirujanos que prestaban servicios de salud con dudosa efectividad y cobertura reducida a los miembros de las cortes de la aristocracia. En el siglo XVIII, se produjo un proceso de gradual ampliación, fortalecimiento y legitimación de la "comunidad médica corporativa", definida como "un conjunto complejo tripartito de médicos, cirujanos y boticarios agrupados en varias Colectividades legales reconocidas"(17).

En 1790, el cirujano Félix Vicq-d'Azyr presentó a la Asamblea Nacional un nuevo plan de organización de la medicina en Fran$\mathrm{Cia}^{(19)}$. La principal marca de esta propuesta era la unificación definitiva entre Medicina y Cirugía, separadas desde la Edad Media, para lo cual los hospitales deberían tener salles d'autopsie y todas las muertes serían confirmadas por anatomistas acreditados. En la interfaz con la educación médica, proponía 
que, no solo la fisiología, sino que todo conocimiento relacionado con enfermedades y su tratamiento debería ser subordinado a la anatomía. De esta forma, establecía, por un lado, una conexión práctica entre clínica, patología y anatomía y, por otro, la importancia de la anatomía comparada en la historia natural. Desgraciadamente, Vicq-d'Azyr no sobrevivió al período del Terror para completar su contribución a la reforma de la medicina ${ }^{(19)}$.

En 1792, Antoine de Fourcroy, médico y farmacéutico heredero intelectual de Vicqd'Azyr, presentó a la Convención una propuesta de sistema de educación superior basado en la "enseñanza libre de las ciencias y de las artes", con foco en la salud. Proponía la reestructuración total de la enseñanza médica a partir de una "medicina en libertad", sustituyendo las facultades de Medicina por écoles de santé destinadas a graduar officiers de santé(20). Farmacoquímica y Administración Pública serían materias fundamentales para una formación profesional polivalente y eficiente. En la dimensión pedagógica, anticipaba una perspectiva integradora de la formación médica, reemplazando la vieja instrucción retórica por didácticas prácticas en laboratorio, basadas en la demostración experimental. Con amplio apoyo de los próceres de la Revolución, el Plan d'Azyr-Fourcroy fue aprobado en febrero de 1794, siendo ampliado a dos escuelas de salud en las provincias, en especial, en Montpellier y en Estrasburgo ${ }^{(20,21)}$.

La primera école de santé, instalada en París, tenía tres clases de alumnos regulares (officiers de santé, chirurgiens, médecins), además de alumnos oyentes (auditeurs libres $)^{(22)}$. La clase de cada estudiante se definía mediante exámenes públicos, diferentes en contenido: anatomía, operaciones y curativos, materia médico-quirúrgica, si el objetivo fuera graduarse en Cirugía; y anatomía y fisiología, campo médico, historia natural de las enfermedades internas y externas, si el candidato se postulaba a la graduación en Medicina ${ }^{(22)}$. La duración de la formación variaba: officier de santé, tres años; chirurgien, cuatro años, médecin, cinco años. Para los graduados que pretendían seguir la carrera médica, además de la aprobación en los exámenes, era necesario elaborar y defender una thèse de doctorat.

El título de officier de santé, elemento clave de la propuesta, enfatizaba una noción práctica de salud positiva, y posiblemente fue bien aceptado en un primer momento porque ayudaba a superar la distinción tradicional entre médicos y cirujanos. El formato institucional reproducía una organización militar, con uniforme y jerarquía de carrera, lo que en teoría facilitaría su aceptación social en una coyuntura de guerra. A la vuelta del siglo, después de tres años de funcionamiento, las écoles de santé ya registraban 4.027 estudiantes; de esos, 406 eran tesistas (thésards) postulantes a Medicina ${ }^{(21)}$. En concreto, el Plan d'Azyr-Fourcroy sufrió retrocesos estratégicos que comprometieron su viabilidad, principalmente, debido al desarrollo de un modelo de corporación profesional orgánicamente articulado con la red política republicana ${ }^{(23)}$.

En 1798, Cabanis presentó al Consejo de los Quinientos un projet de résolution para la organización de la profesión médica y el cuidado en salud, basado en una teoría de economía política capaz de justificar intervenciones profundas en la incipiente red social de asistencia en salud ${ }^{(24)}$. Más que control de mercado y control social, la naciente República debería organizar y proveer dispositivos de legislación y medios de control del Estado, con el objetivo de regular la producción y utilización de esos procesos y productos, sobre todo en espacios sociales estratégicos como aquellos donde se realiza la práctica médica. Para avanzar en estas medidas, priorizaba dos soluciones: la acreditación oficial del ejercicio profesional y el reconocimiento de los officiers de santé, profesionales prácticos formados en momentos de gran carencia de mé$\operatorname{dicos}^{(24)}$. Foucault ${ }^{(18)}$ informa que la propuesta de Cabanis fue luego rechazada. Todavía, la regulación de la práctica médica de 1802, realizada por el Consulado de Bonaparte y mantenida aún por medio siglo, incorporaba prácticamente todas las proposiciones de Cabanis. En ese momento, de modo pionero, se establecía la tutela del Estado y el concepto de ejercicio ilegal de la profesión, en la medida en que cualquier ciudadano no licenciado, 
que se arriesgara a practicar la medicina, incurriría en penalidades, de multas a prisión ${ }^{(18)}$.

Una vez confirmado el fracaso del Plan d'Azyr-Fourcroy, se abrió espacio para el resurgimiento de facultades, reforzadas por sociedades y corporaciones fuertemente empoderadas, ahora independientes y sin el control central de academias. Aprovechando su posición política en el Consejo de los Quinientos y después en el Senado, Cabanis se fortaleció como cuadro intelectual importante en ese proceso. Así, bajo su liderazgo, se registra la elaboración, proposición y aprobación de un conjunto de planteos que, con cierto distanciamiento histórico, por una cuestión de justicia intelectual, fue designado como reforma Cabanis ${ }^{(25)}$.

\section{LA REFORMA CABANIS}

Como plan general, la reforma Cabanis incorporaba propuestas parciales, cada una de ellas de naturaleza coyuntural y cargando simultáneamente un sentido político y un fuerte sesgo práctico. En términos políticos, articulaba diversos movimientos de reforma de la instrucción pública y de organización del cuidado de la salud que habían sido formulados en el período posrevolucionario, inspirados en el racionalismo cartesiano y sus desdoblamientos. Por un lado, defendía una red pública de educación gratuita en todos los niveles, con acceso universal, compuesta, en la esfera de la educación superior, por una gran diversidad de órganos aislados y especializados de formación profesional. Por otro lado, proponía la superación de la universidad como concepto y como organismo coordinador, delineando una estructura institucional descentralizada, basada en facultades y escuelas aisladas.

Como proyecto pragmático, sintetizado en el Coup d'oeil sur les révolutions et réforme de la Médecine ${ }^{(13)}$, la reforma Cabanis se manifiesta bajo la forma de propuestas concretas en distintas dimensiones y etapas de reforma de la medicina y de la enseñanza médica. Comprende cinco elementos estructurales: a) habilitación profesional mediante certificación académica, b) un modo peculiar de organización institucional; c) redefinición de espacios laicos de enseñanzaaprendizaje; d) nueva estructura curricular; e) filosofía pedagógica innovadora. Veamos cada uno de estos puntos.

\section{Habilitación profesional mediante certificación académica}

Inicialmente enfocándose en la práctica médica, la reforma Cabanis introdujo el concepto de "licenciatura", dispositivo legalmente habilitador del ejercicio profesional. La autorización de la práctica médico-quirúrgica, mediante certificación otorgada por una institución de enseñanza superior acreditada, permitiría mayor control del Estado sobre una esfera central de la vida civil necesaria para modernizar las relaciones de trabajo y proteger la salud de las personas. En este régimen, la institución de enseñanza superior tiene la responsabilidad de emitir diplomas formalmente reconocidos como válidos $y$, por lo tanto, habilitadores para la práctica profesional. El carácter de instrumento regulatorio del diploma médico se extendió rápidamente a otras profesiones que implicaban, por un lado, responsabilidad civil y relevancia social y, por otro lado, acceso a mercados de trabajo de impacto económico prioritario y mayor acumulación de capital político ${ }^{(18)}$.

\section{Un modo peculiar de organización institucional}

La enseñanza médica debería realizarse en unidades independientes, con la autonomía posible ante el Estado regulador y el organismo universitario central, en aspectos pedagógicos de la práctica académica y en la conducción de actividades de formación. En el modelo cabanisiano de educación superior, los estudiantes entrarían directamente en facultades o escuelas médicas. Como estructura interna, las facultades serían organizadas en el régimen de cátedra, definida como la 
unidad productiva de la instrucción, gestionada académica y administrativamente por un único liderazgo intelectual capaz de dominar todo el conocimiento sobre esa materia: el catedrático. El perfil de competencias profesionales definido en ese modelo configuraba una práctica diagnóstica de base semiológica, orientada por conocimientos científicos naturalistas, confirmada por respuestas terapéuticas y correlaciones anatomo-clínicas.

\section{Redefinición de espacios laicos de enseñanza-aprendizaje}

Para modernizar el modelo de formación de los profesionales de la salud, sería necesario redefinir espacios de aprendizaje adecuados a una nueva práctica clínica fundada en el conocimiento nosológico sistemático, a partir del proceso de laicización de los hospitales, transformándolos en instituciones de cuidado en salud ${ }^{(18)}$. Así, en vez de la relación maestro-aprendiz, propia de la formación clínica prácticamente artesanal que era propiciada por el acompañamiento de médicos particulares en visitas domiciliarias, la reforma Cabanis tornaría obligatoria la instrucción profesional en hospitales, con la invención del internado (internat des hôpitaux) que, después evolucionó hacia el concepto moderno de residencia médica. Con la unificación entre Medicina y Cirugía, se recomendó la implantación de teatros quirúrgicos, además de las salles d'autopsie preconizadas por Vicq-d'Azyr ${ }^{(19)}$.

\section{Nueva estructura curricular}

En la nueva estructura curricular, el conocimiento fue descompuesto en sus partes constitutivas: las disciplinas, en la dimensión cognitiva, que correspondieron al concepto de cátedra, en la dimensión de organización institucional. En ese sentido, la disciplina comprendía el conjunto de saberes y conocimientos de acceso restringido a los discípulos de un catedrático. En el caso del conocimiento operativo del saber médico, foco original de la reforma Cabanis, las disciplinas deberían ser, a efectos de la estructura curricular, agrupadas en historia natural (ciencias básicas), materia médica (ciencias clínicas y terapéuticas) y ciencias morales ${ }^{(13)}$. Además, principalmente con vistas a la incorporación de la práctica quirúrgica experimental, la enseñanza de medicina humana necesitaba ser articulada a la enseñanza de la medicina veterinaria. La principal consecuencia de este modelo de organización curricular disciplinaria sobre los modos de cuidado en salud fue la fragmentación de la práctica (inicialmente quirúrgica y después clínica) en especialidades médicas.

\section{Filosofía pedagógica innovadora}

La reforma Cabanis implicaba una estrategia pedagógica innovadora, denominada por él como "método analítico de enseñanza", equivalente al análisis racional de los ideólo$\operatorname{gos}^{(13)}$. El conocimiento científico solamente sería validado si era descompuesto y recompuesto por el orden lineal cartesiano, de la materia más simple a la más compleja, en una operación de exhaustividad propia del enciclopedismo. Así, la pedagogía analítica correspondería a la fragmentación, análisis y posterior ordenación de bloques de contenidos, modos de práctica y, principalmente, conocimientos memorizados, almacenados, codificados y clasificados según las doctrinas científicas vigentes. Esto requeriría la superación de la retórica como modelo pedagógico, con el consiguiente abandono del latín como lenguaje técnico, codificado como elemento de diferenciación y elitización de la corporación profesional.

\section{La reforma Cabanis y la Idéologie}

La reforma Cabanis se instituye en la interfaz entre la clínica, la política y la didáctica, e implica también un cambio crítico en las estrategias pedagógicas de formación en el campo de la salud, a partir de la fragmentación sistemática tanto del proceso de formación como 
del objeto de enseñanza-aprendizaje. El modelo de formación resultante se basaba en una apología de la disciplinariedad como modo de organización y catalogación del conocimiento humano en una estructura racional y sistemática. En ese espíritu, tiene la intención de contribuir a la nueva pedagogía necesaria para una reforma de la enseñanza médica, a su vez imprescindible para la sostenibilidad de la revolución realizada en los sistemas de salud de Francia. Así, la reforma Cabanis se integra al proyecto de los ideólogos de crear una nueva epistemología para un nuevo paradigma de ciencia comprometida políticamente con el progreso humano, en el umbral del iluminismo hacia la modernidad ${ }^{(9,14,15)}$.

La triple reforma (política, práctica y pedagógica) protagonizada por Cabanis puede ser resaltada como un movimiento crucial de articulación entre una estructura ideológica (en el sentido propio de los ideólogos) fundada en disciplinas científicas básicas para la formación médica y un contexto de prácticas profesionales especializadas y exclusivas. La principal consecuencia de este proceso, al menos en la esfera de influencia del modelo francés de educación, fue la hegemonía de una formación disciplinaria fragmentada en una universidad que, si bien antes era la casa de las ciencias, las artes y la cultura en una perspectiva amplia e integradora, se hizo una institución vocacional, promotora de formación técnica especializada orgánica al nuevo modo de producción fundado en la producción industrial masificada. Una primera hipótesis atribuye el surgimiento de la especialización profesional a la diferenciación académica, con ocurrencia de subdivisiones entre disciplinas científicas ${ }^{(26)}$. Weisz ${ }^{(27)}$ propone una tendencia inversa: la especialización se habría iniciado en París entre 1830 y 1850 , sobre todo, en el campo profesional de la salud, en el proceso de acomodación entre espacios de formación profesional, determinada por un mercado de trabajo ya de inicio competitivo, y de ahí a la consolidación de trayectorias curriculares especializadas.

\section{Difusión del modelo cabanisiano}

En apenas seis años, entre 1794 y 1800, ideas, soluciones y acciones que conformaron la reforma Cabanis, se concretaron primero en la reorganización de la Facultad de Medicina de París, con activa participación de Cabanis, entre otros contemporáneos ${ }^{(18,25)}$. De hecho, la reforma de la enseñanza médica sirvió como modelo para todo el sistema de enseñanza superior francés, en aquella época estructurado como una red de instituciones aisladas, como la Escuela Politécnica y la Escuela Normal Superior, exteriores a la universidad, que tenían por objetivo atender a las exigencias de la revolución industrial y a las demandas de cuadros dirigentes para la burocracia estatal ${ }^{(6,7,28)}$. El nuevo régimen podría contar con una educación superior dominada por nuevas profesiones legitimadas y controladas por el Estado burgués, sin perder los atractivos de la tradición académica que, en cierta medida, habían sido proscritos y que serían rápidamente restaurados posteriormente por el Consulado y el Imperio. La reforma Cabanis resultó finalmente en facultades de medicina dotadas de autonomía y capacidad para decidir de modo independiente sobre normas, disciplinas y cursos, desplazando a la universidad como eje institucional de la educación superior en el orden social del capitalismo emergente en el advenimiento de la modernidad ${ }^{(25)}$.

Extrapolando el campo de la salud, la reforma Cabanis fue incorporada a la reforma educativa bonapartista que estableció las bases institucionales del sistema de enseñanza superior implantado en Francia, durante el siglo XIX ${ }^{(25)}$. En esa época, libros, revistas, compendios y tratados científicos y filosóficos producidos en Francia eran exportados y luego traducidos a otras lenguas. La obra de Cabanis y su reforma no fueron excepción, habiendo sido ampliamente difundida en toda Europa, desde los primeros años del siglo XIX. El Coup d'oeil, por ejemplo, fue traducido y publicado en inglés dos años después de su primera edición. Su primera traducción en castellano fue publicada en Madrid, en 1820, bajo el título Compendio histórico de las revoluciones y reforma de la Medicina ${ }^{(29)}$. 


\section{LA PRESENCIA DE CABANIS EN ARGENTINA}

En 1623, en la gobernación del Río de la Plata y Paraguay, colonia española que, tras la independencia, se convertiría primero en las Provincias Unidas del Río de la Plata y a posteriori en la República Argentina, los Estados Pontificios autorizaron la fundación de la Universidad de Córdoba en el Colegio Máximo de la Compañía de Jesús en la ciudad de Córdoba, centro de la gobernación del Tucumán. Tomando la Universidad de Salamanca como modelo institucional, la escolástica medieval tardía como referencia doctrinal y la Ratio Studiorum como referencial pedagógico, la Universidad de Córdoba fue la principal institución de educación superior en el Río de la Plata durante casi dos $\operatorname{siglos}^{(30)}$. Con la apertura de la primera cátedra de Derecho Civil en 1791, esa casa de enseñanza fue refundada en 1808, manteniendo su estructura curricular canónica y sin interrumpir la enseñanza de Teología, incluso después de la expulsión de los Jesuitas por la Corona española en 1767.

Terminado el monopolio educacional de la Compañía de Jesús, en la capital de Buenos Aires, en la víspera de la independencia de las Provincias Unidas del Río de la Plata en julio de 1816, se fundaron tres instituciones de educación superior: el Instituto Médico-Militar, la Academia de Matemáticas y la Academia de Jurisprudencia ${ }^{(31)}$. En agosto de 1821, un decreto del gobierno provincial porteño creó la Universidad de Buenos Aires como órgano de coordinación y supervisión de actividades educativas en la provincia de Buenos Aires, cuyo proponente y primer rector fue el presbítero Antonio Sáenz.

La instauración de la universidad porteña se vinculó fuertemente a la construcción de un régimen político estable que se configuró en 1821 a partir de una articulación entre la dirigencia porteña y los sectores rurales en ascenso de la provincia de Buenos Aires, asociación que suele conocerse con el nombre de grupo rivadaviano o movimiento rivadaviano. El grupo rivadaviano estaba conformado a partir de una identidad sumamente ambivalente, constituida en dos niveles: uno, en torno a los miembros de la nueva legislatura y el ministerio de gobierno ejercido por Bernardino Rivadavia; el otro, que involucraba una serie de sectores más amplios y en los que aparecía la lógica de la política de círculo o de facción ${ }^{(32)}$. Bajo la gestión del gobernador Martín Rodríguez surgió un inusitado diseño del espacio público: el modelo republicano y representativo de gobierno asentado en nuevos mecanismos institucionales. En la provincia de Buenos Aires (a diferencia del resto de las provincias) se consolidó informalmente un sistema de poder legislativo y un mecanismo de elección de sus integrantes. En 1821 se dictó la ley electoral y el derecho al voto "universal" que incorporaba a la campaña bonaerense. El derecho al sufragio junto a un régimen de elección directa de diputados a la Sala de Representantes -poder legislativo provincial creado en 1820 y encargado de designar al gobernador- causó una nueva concepción de la relación entre gobernantes y gobernados.

Paralelamente a la conformación del régimen político, Bernardino Rivadavia, ministro de gobierno de Rodríguez, implementó un abanico de reformas en el ámbito político, institucional, económico, eclesiástico, educativo, cultural y militar ${ }^{(33)}$. El objetivo de estas reformas en su conjunto era terminar drásticamente con la herencia de la estructura colonial y "modernizar" las prácticas y el imaginario político. Así se observa cómo la reforma Cabanis se ligó a este plan reformista rivadaviano que buscó conformar un tipo de ciudadano basado en una matriz "republicana-ilustrada" en consonancia con el nuevo orden político. Para ello, la universidad aparecía como un espacio clave en la formación de los cuadros profesionales y pensadores como Destutt de Tracy y Cabanis sus referencias centrales. Como vimos anteriormente, se trata del modelo bonapartista de universidad imperial, según lo descrito por Buchbinder ${ }^{(30)}$ :

La casa de estudios porteña fundada por iniciativa de Sáenz administraba todo el 
sistema educativo que funcionaba en la órbita del Estado provincial, incluso la enseñanza básica, y su creación debe entenderse en el marco de un programa más amplio de promoción de todo el sistema de educación pública.

Rápidamente se constató que, si el concepto estricto de universidad nominal no se mantenía ni siquiera en su país de origen después de la caída del emperador Napoleón I en 1815, no había por qué introducirlo en Buenos Aires. En ese momento, los institutos y academias existentes fueron incorporados a la nueva institución universitaria, componiendo una estructura de cátedras organizadas en seis departamentos que revelaban equivalencia con las facultades superiores clásicas (Primeras Letras, Estudios Preparatorios, Medicina, Jurisprudencia, y Ciencias Sagradas), además del Departamento de Ciencias Exactas ${ }^{(31)}$. Pero también se introdujeron modificaciones importantes como la inclusión de nuevas asignaturas y docentes. Las reformas más importantes ocurrieron en el Departamento de Estudios Preparatorios, en el que se crearon nuevas cátedras, como la de Química Experimental a cargo de Manuel Moreno (desde abril de 1822 hasta marzo de 1828), la de Física Experimental, a cargo del médico italiano Pedro Carta Molina (desde abril de 1826 hasta noviembre de 1827) y la de Ideología, a cargo del profesor Juan Manuel Fernández de Agüero (desde marzo de 1822 hasta diciembre de 1823). Asimismo, desde 1821, Avelino Díaz [18001831] que se había formado en la Academia de Matemáticas con Lanz y Senillosa, dictó el curso de Físico-Matemáticas, de dos años de duración.

\section{La Idéologie en la Universidad de Buenos Aires}

Cabe indicar que un vector importante de difusión de la Idéologie en Buenos Aires se produjo a partir de la relación personal entre Desttut de Tracy y Rivadavia entablada por aquellos años. Varias cartas acreditan este intercambio, que remite a un conocimiento previo y personal producto de la estadía en Francia de Rivadavia en dos ocasiones (18141820 y $1824-1825)$

La mayoría de las cartas están editadas y traducidas por Ricardo Picirrilli ${ }^{(34)}$. Otras, sin editar ni traducir fueron localizadas en el Museo Mitre de la República Argentina. En una de las cartas, Destutt de Tracy escribe a Rivadavia que: "A la verdad, es una bella institución la de una cátedra de Economía política y otra de Ideología. En cuanto a esta última yo me considero feliz, si mi obra puede ser de alguna utilidad, entre tanto no aparezca otra mejor" (Carta de Destutt de Tracy a Bernardino Rivadavia, París, 18 de noviembre de 1822; en el Museo Mitre, Colección Correspondencia Hombres Públicos Argentinos, ref. 1C22C16, Nro. 1, folio 15925).

Otra vía de difusión se produjo a través de la prensa: diversos registros y referencias en periódicos de la época lo constatan tales como El Centinela (1822), La Abeja Argentina (1822-1823), El Argos de Buenos Aires y Avisador Mercantil (1824) y El Correo Político y Mercantil de las Provincias Unidas del Río de la Plata (1827) ${ }^{(35)}$. En ese proceso, indicios numerosos e intrigantes demuestran la intensa y sorprendente presencia del movimiento de los ideólogos franceses en especial en el marco de las cátedras de la flamante universidad $^{(36)}$.

La cátedra de Lógica, Metafísica y Retórica fue la primera de la Universidad de Buenos Aires. Su fundador, el sacerdote Juan Manuel Fernández de Agüero [1772-1840], religioso español, en su obra Principios de ideología elemental abstractiva y oratoria (publicada en 1824) defendía la inusitada combinación entre filosofía escolástica y materialismo sensualista de Condillac. Al asumir sus deberes en la universidad, renombró su materia como cátedra de Ideología, ampliando su alcance para un vasto conjunto de cursos en el campo de las humanidades (desde filosofía, ética y lógica, a la historia, economía política y filología), difundiendo el pensamiento de Desttut de Tracy y de Cabanis. Fernández de Agüero $^{(37)}$ sigue de cerca a Desttut de Tracy y a Cabanis cuando sostiene que: 
Así pues lo físico y lo moral se confunden en su origen, o por mejor decir lo moral no es otra cosa que lo físico considerado bajo ciertos puntos de vista más particulares. Para la completa inteligencia de esto bastará observar que la vida es un tejido de movimientos ejecutados a virtud de las impresiones recibidas por los diferentes órganos; y que las operaciones del alma o del espíritu resultan igualmente de los movimientos ejecutados por el órgano cerebral; y sus alteraciones de impresiones recibidas y comunicadas por las extremidades sencientes de los nervios en las diferentes partes, o excitadas en este órgano por medios que parecen obrar inmediatamente sobre él.

Uno de los giros renovadores que se encuentra presente en el pensamiento traciano fue la inclusión de la ideología dentro de la zoología. De hecho, la subordinación de la Ideología como rama de la Zoología le permite generar el marco previo en la argumentación de uno de los puntos más álgidos de su teoría: la no existencia de una distinción cualitativa entre el hombre y el mundo animal. Destutt de Tracy indica:

\section{Uno solo tiene un conocimiento incom- pleto de un animal, si uno no conoce sus facultades intelectuales. La Ideología es una parte de la Zoología, y es, sobre todo, en el hombre que esta parte tiene impor- tancia y merece ser profundizada. ${ }^{(11)}$}

El catedrático indica que se debe "escuchar a los fisiologistas y abandonar a los teólogos $^{\prime \prime(37)}$. Por estas y otras razones, el rector Sáenz abrió -en las instancias administrativas y eclesiásticas- varios procesos contra Fernández de Agüero, acusado de prácticas escandalosas y de herejía ${ }^{(38)}$.

La situación incierta sobre la conclusión de la guerra con Brasil, la presión de los nuevos grupos rurales para la obtención de más cargos públicos, la oposición de sectores reacios a los cambios introducidos, como el clero y el ejército, la reacción de las provincias ante la Constitución unitaria de 1826, entre otras cuestiones, motivaron la caída de la administración rivadaviana hacia 1827. En Buenos Aires, Manuel Dorrego fue elegido gobernador y con él se introdujeron los principios del federalismo en la escena provincial. No obstante, fue destituido rápidamente por la invasión llevada a cabo por el general unitario Juan Lavalle. Con la muerte de Dorrego, en manos de Lavalle, se generó una crisis política que posibilitó el ascenso de Juan Manuel de Rosas a la gobernación de la provincia.

De las características principales del sistema político rosista podríamos destacar la continuidad del ejecutivo y la centralización del poder. Bajo el régimen rosista, la sociedad se polarizó por el nivel de adhesión o rechazo político con respecto de la gestión de gobierno. Pero cabe indicar que no se desarticuló el andamiaje republicano y representativo introducido por las reformas rivadavianas, sino que fue reordenado bajo otra modalidad $^{(39)}$. Asimismo, durante la primera y segunda gobernación de Rosas se produjo una activa participación de los sectores populares y alta politización del conjunto social ${ }^{(40)}$. La beligerancia permanente contra todos los intentos de oposición, sumada a las guerras entabladas con potencias extranjeras, generaron una situación de constante inestabilidad política $^{(41)}$.

En este entramado particular se insertó la vida intelectual y académica. El proceso de politización de la sociedad, sumado a las guerras internas y externas a lo largo del período, tuvo un impacto significativo en la universidad porteña, en cuyos recintos se experimentaron una serie de divisiones, conflictos y opiniones encontradas. Cabe indicar que la institución siguió funcionando pero de forma muy limitada dada la escasez de estudiantes, las presiones facciosas, las renuncias y despidos de profesores, y la falta de financiamiento por parte del gobierno ${ }^{(30)}$. A pesar de esta precariedad institucional, los saberes de la Idéologie siguieron difundiéndose en las aulas a través del impulso dado por Diego Alcorta, médico que se haría cargo de la Cátedra de Ideología entre 1828 y 1842. 


\section{Cabanis, Alcorta y la enseñanza médica}

En 1827, Diego Alcorta finalizaba sus estudios y recibía el título de doctor en Medicina con un trabajo titulado Disertación sobre la manía aguda, considerado el primer documento científico de la psiquiatría local, fuertemente influenciado por Pinel y Cabanis, particularmente por sus respectivos estudios sobre la articulación nosología, fisiología y patología y sobre las relaciones mentecuerpo $^{(42)}$. Al igual que Cabanis, Alcorta sostiene que los órganos imprimen las sensaciones de los objetos y, por lo tanto, son los que producen las ideas y los sentimientos del hombre, y entiende que las enfermedades mentales o morales son producto de las alteraciones orgánicas ${ }^{(12)}$. Al respecto expresa que "se ha descubierto por un gran número de disecciones comparadas que a sus enfermedades corresponden frecuentemente alteraciones en las facultades morales" ${ }^{\prime(43)}$.

$\mathrm{Al}$ esquema de las sensaciones que extrae Fernández de Agüero de Destutt de Tracy, Alcorta incorpora el de Cabanis, cuando indica que: "...las distintas épocas de la vida vienen acompañadas del desarrollo particular de algunas de las facultades intelectuales, y de ciertos sentimientos interiores nacidos del estado actual de los órganos de la economía"(43). En este sentido, también manifiesta que "...toda impresión demasiado fuerte, tanto física como moral, puede determinar la manía; pero para ello es necesaria una predisposición individual que es originaria o es ocasionada por la educación, la edad, la manera de vivir, el sexo, etc." ${ }^{\prime(43)}$. El funcionamiento orgánico de los sujetos con respecto a los comportamientos que experimentan queda articulado: las enfermedades mentales atraviesan el aspecto físico y moral en un mismo nivel ${ }^{(12)}$. Por otro lado, Alcorta confecciona una guía para el dictado de sus clases denominada Lecciones de filosofía, obra editada en $1835^{(44)}$. Dividió el manual en tres partes: Lógica, Metafísica y Retórica. Las nociones centrales de Cabanis pueden identificarse a lo largo de las Lecciones.

En cuanto a la enseñanza médica, la actividad docente del departamento de Ciencias de la Medicina se inauguró en 1822 con un plan de estudios de cuatro años de duración, que debía ser desarrollado por tres profesores: Instituciones médicas, Juan Antonio Fernández; Instituciones quirúrgicas, Cosme Francisco Argerich y Clínica médica y quirúrgica, Francisco de Paula Rivero. En este cuadro de cátedras puede observarse cómo se buscó propiciar una unión más cercana entre la medicina y la cirugía, siguiendo el modelo propuesto por Cabanis. El plan de estudios proyectado primitivamente era más amplio pero los problemas económicos obligaron a cercenarlo (en el Archivo General de la Nación de la República Argentina, Sala X, Tribunal de Medicina, legajo 6-2-2, figuran dos de esos planes: uno de siete cátedras y el otro de seis años y dieciocho materias más un curso práctico anual de disección). Pero hubo sin embargo un avance importante: en 1822 se inauguró en el Departamento de Medicina la primera sala de disección anatómica. Aclaremos que una sala anatómica es un espacio diseñado para las demostraciones y la enseñanza. Allí, se practicaba la disección que consistía en la división en partes de una planta, un animal o un ser humano muertos para examinarlos y estudiar sus órganos. El primer anfiteatro anatómico se construyó en 1594 en la Universidad de Padua ${ }^{(45)}$.

Programada la enseñanza y creado un proceso tendiente a la habilitación de una carrera médica licenciada y diplomada en los estudios superiores, quedaba por direccionar y controlar mejor las actividades de los médicos en tanto prácticas legales, oficiales e institucionalizadas. Con esa finalidad, se creó el Tribunal de Medicina, cuyas atribuciones y obligaciones figuran en forma detallada en el Reglamento conocido como "Arreglo de la Medicina" del 9 de abril de 1822 (Archivo General de la Nación, Sala X, Tribunal de Medicina, legajo 6-2-2). Los dos primeros artículos de los diez que comprendía el Reglamento están dedicados a especificar sus funciones, que resultaban ser similares a las que tenía el anterior tribunal. Efectivamente, se ocuparía de dictar las medidas profilácticas en casos de contagio o infecciones, de inspeccionar los establecimientos asistenciales, 
de proponer facultativos para los diferentes destinos. Tenía, además, que llevar un libro de registro de títulos, en los que se asentarían todas las reválidas que se efectuasen. El Tribunal estaba facultado no solo para dar dictamen a los jueces en caso de medicina legal, sino para juzgar para sí mismo los abusos, la impericia médica y los casos de curanderismo. Estas funciones de control que ejercía el Tribunal de Medicina no solo se manifestaron en decretos o reglamentos, sino que también fueron efectivas para alcanzar y regular la práctica profesional como lo indicaba un viajero inglés anónimo ${ }^{(46)}$ : “Una entidad científica, creada hace poco tiempo, examina las condiciones de los médicos locales, haciendo preguntas que pondrían en aprietos hasta al mismo Esculapio".

Los médicos aportan su concurso y su caución a esta dinámica de apropiación de ideas referidas a la salud y la enfermedad, al tiempo que encuentran allí un importante vector de promoción. No es sorprendente que la incipiente medicina rioplatense haya puesto su atención en los saberes de la Idéologie que le ofrecían, por un lado, una teoría fisiológica que conectaba las funciones de la mente con el cuerpo y, por otro, la posibilidad de legitimarse como ciencia moderna sin la intervención eclesiástica en estas materias. A partir de la exploración de este problema se observó la creciente importancia que fue ganando el médico en el espacio cultural porteño. Hacia la década de 1830 la medicina comenzaba a considerarse una actividad científica, académica y profesional y esta modernización, que a su vez implicaba salirse de los rasgos "supersticiosos" de la época colonial, tuvo una estrecha relación con la difusión en Buenos Aires de los saberes de la Idéologie y en especial los de Cabanis.

De esta manera, por más de una década, entre 1824 y 1835 , ese pionero y peculiar sistema departamental se amplió, lo que resultó en la multiplicación de cátedras, con gran fragmentación, frecuentes duplicidades y duras disputas territoriales, generando sucesivas crisis. En este proceso, la demanda por la enseñanza superior disminuyó, la evasión aumentó, el Departamento de Ciencias
Sagradas fue prácticamente desactivado por falta de estudiantes y el Departamento de Ciencias Exactas terminó dividido en tres áreas: Matemáticas Puras, Matemáticas Aplicadas e Historia Natural. Las crisis se agudizaron hasta que, en 1852, culminaron con la salida de la universidad de de una gran parte de los profesores del Departamento de Medicina para constituir una Facultad de Medicina autónoma $^{(31)}$. En 1860, la proto-universidad porteña se limitaba a los departamentos de Estudios Preparatorios y de Jurisprudencia ${ }^{(30)}$.

\section{Diversidad en la universidad argentina}

Recién en marzo de 1874, tras un intenso trabajo político del rector Juan María Gutiérrez, la Universidad de Buenos Aires fue reinstituida mediante decreto del Poder Ejecutivo provincial ${ }^{(30,31)}$. La institución reformada contaba con un régimen de gobierno descentralizado y una estructura organizativa compuesta por cinco facultades: Humanidades y Filosofía, Derecho y Ciencias Sociales, Ciencias Médicas (reincorporando la Facultad de Medicina), Ciencias FísicoNaturales, Matemáticas. Los efectos inmediatos de esta reforma son comentados por Buchbinder ${ }^{(30)}$ :

La Universidad dejó de ser concebida entonces como un organismo unitario y se transformó en una suerte de federación de facultades. Dicho decreto sentó las bases para un nuevo régimen de gobierno de la institución que se mantendría hasta los primeros años del siglo. Se estableció entonces que el gobierno de la casa de estudios estaría presidido por un Consejo Superior encabezado por el rector e integrado por los decanos y dos delegados por cada una de las facultades. Éstas serían gobernadas ahora por academias responsables, además, de la formulación de sus reglamentos internos y planes de estudio.

Con un proyecto elaborado por el senador Joaquín González, el Congreso de la 
República Argentina aprobó la creación de la Universidad Nacional de La Plata, a partir de una pequeña universidad provincial que había sido fundada en la provincia de Buenos Aires, en 1897. González fue designado su primer rector en 1906. La nueva universidad tenía como objetivo "formar docentes para escuelas y colegios, científicos para el desarrollo de las ciencias y las industrias, y filósofos para la conducción de la República"(30). El plan institucional tenía como referencia el sistema anglosajón de colleges, en los moldes de Oxford y Cambridge, y el modelo humboldtiano alemán con museos, laboratorios y observatorios para la formación científica avanzada. Contaba con un Colegio Nacional para acoger a todos los nuevos estudiantes, algunos en residencia en el campus, en bachilleres de formación general. La formación científica y profesional específica se daba en cursos universitarios ofrecidos en cinco grandes facultades: Ciencias Naturales; Ciencias Físicas, Matemáticas y Astronómicas; Agronomía y Veterinaria; Ciencias Jurídicas y Sociales; Ciencias de la Educación. En las dos primeras décadas de funcionamiento, gracias al liderazgo político e intelectual del senador González, aprovechando un período de gran desarrollo económico basado en la exportación de carne y derivados, la Universidad Nacional de la Plata contó con apoyo político, institucional y presupuestario suficiente para transformarlo en referencia internacional.

En contraste con la situación de las otras universidades argentinas en el período, el inicio de esta peculiar universidad es narrado por Buchbinder ${ }^{(30)}$ :

El intercambio académico con centros universitarios europeos fue otra de las bases de la política que se implementó en la institución. [...] Varios científicos y académicos europeos y norteamericanos fueron invitados a conocer la Universidad y a dictar cursos y conferencias. El programa de extensión contemplaba la visita sistemática del público a sus bibliotecas, museos y laboratorios, la asistencia de sus profesores a escuelas y colegios, una ambiciosa política editorial y, sobre todo, un plan de conferencias públicas llevado a cabo por invitados especiales y por las autoridades y docentes de la casa de estudios.

En la práctica, el Plan González sufrió retrocesos, derrotas institucionales y sabotaje de todo orden, debido a la reacción de un sistema corporativo profesional y académico articulado con la coyuntura política nacional. A pesar de configurar un modelo de reforma de la educación superior, demostrado en su efectividad inmediata y viabilizada políticamente, no fue acogido en ningún ámbito universitario fuera de la provincia.

El alienista y filósofo Alejandro Korn, intelectual crítico de la historia cultural de Argentina, considera este modelo de universidad como un proyecto desde el principio condenado al fracaso porque era un intento de trasplante institucional realizado por una generación de "positivistas universitarios", impuesto sobre un contexto de desarrollo colonizado que revela "una desconexión entre la Universidad y la cultura nacional"(47). Se llegó a afirmar que poco después de su implantación la Universidad Nacional de La Plata se había transformado en un mal arreglo de la antigua Universidad situada a una hora de distancia. Y luego, completa Buchbinder ${ }^{(30)}$ :

...hacia finales de la década de 1910 las
tensiones y los problemas de las grandes
universidades se reproducían también
en la casa de estudios platense. [...] Sus
institutos científicos generaron escaso
interés entre sus estudiantes potencia-
les, que optaron por las carreras tradi-
cionales como en las casas de estudio
más antiguas y buscaron el ansiado y
cotizado título que permitía el ejercicio
liberal de la profesión.

Así, a comienzos del siglo XX, Argentina pasaba por una situación al menos curiosa: contaba simultáneamente con una universidad medieval, una universidad humboldtiana y una no-universidad bonapartista. De hecho, cada una de sus principales instituciones de 
enseñanza superior representaba uno de los modelos de universidad establecidos en la historia occidental: la Universidad de Córdoba (arcana, medieval, religiosa, escolástica); la Universidad de Buenos Aires (bonapartista, laica, profesionalizante, liberal); la Universidad Nacional de la Plata (científica, vocacional, humboldtiana, articulada con el sistema productivo, al menos como proyecto $)^{(30,31)}$. En 1918, los estudiantes de la Universidad de Córdoba, apoyados por una parte reducida del cuerpo docente, iniciaron una huelga general que se transformó en una rebelión pública, en lucha contra la rigidez pedagógica y el anacronismo de la formación, reivindicando autonomía institucional, participación y más amplia gobernanza de la universidad $^{(48)}$. La dura represión política que siguió, desafiada por la resistencia de la comunidad universitaria y por la solidaridad de grupos movilizados de la sociedad, resultó en una profunda crisis de autoridad, solo resuelta con cambios académicos y concesiones políticas en todo el sistema universitario argentino.

La Reforma Universitaria de 1918, celebrada como importante marco político en la historia de la educación latinoamericana, hoy puede ser evaluada más críticamente como incorporación activa (sin embargo, extemporánea) de valores democráticos y seculares de la revolución burguesa del cambio del siglo XIX. Eso pasó en un contexto de ascenso de una clase media orgánica al modelo de desarrollo nacional, en curso a principios del siglo $X X^{(3)}$. En esta reforma, se confirma la hegemonía del modelo establecido en la Universidad de Buenos Aires, fuertemente influenciado por la Idéologie en su inicio y, en su implantación, dedicado a la enseñanza profesional en detrimento de la formación académica de base científica ${ }^{(30,31)}$. En ese régimen, no cuesta recordar, que la universidad tenía un papel meramente nominal, en la medida en que se concedía enorme poder y autonomía a las facultades, particularmente Medicina y Derecho, formadoras de las profesiones liberales.

\section{COMENTARIOS FINALES}

Sin duda, Georges Cabanis fue un hombre de su tiempo, representante de un pensamiento político que hoy se puede apreciar como conservador; sin embargo, la reforma Cabanis fue revolucionaria, en su tiempo, hace más de dos siglos. En efecto, Cabanis fue uno de los más importantes líderes intelectuales y políticos que estuvieron activos en las articulaciones que acomodaron los avances de la Revolución francesa de 1789, en un proceso de recuperación de los valores de la tradición cultural europea mediterránea. Su opción política durante la reconstrucción de Francia fue pragmática, posicionándose al lado de los moderados durante el régimen posrevolucionario; pero posteriormente asumió una postura valientemente crítica, perfilándose entre los opositores desencantados con el autoritarismo bonapartista ${ }^{(10,14,15)}$. No obstante, el modelo educativo concebido por él y sus pares del Círculo de Auteuil fue totalmente absorbido en la reforma educativa bonapartista que estableció las bases del sistema de enseñanza superior y profesional implantado en Francia a lo largo del siglo XIX, extrapolando el ámbito restringido de la educación superior en salud. La demostración de cómo una reforma sectorial de la enseñanza médica resulta en cambios profundos en la educación superior en general no se aplica solo a la coyuntura posrevolucionaria en Francia: un siglo más tarde, la reforma de la enseñanza médica realizada por Flexner terminó produciendo amplia renovación en el sistema universitario estadounidense ${ }^{(49)}$.

La aproximación inicial al proceso de constitución del modelo de enseñanza superior predominante en la Argentina, presentada en este ensayo, confirma la presencia orgánica de efectos indirectos de la reforma Cabanis. La cuestión planteada ahora no es si las raíces de la educación superior en salud en América Latina se pueden remontar al modelo francés de formación, sino en qué medida resultan de ese proceso histórico, mediada por la gravitación de la Idéologie (Argentina) y del positivismo (Brasil). La evaluación de la hipótesis de que 
la universidad latinoamericana conserva las marcas de la reforma Cabanis implica verificar la presencia concreta y efectiva, en los establecimientos, programas o cursos de formación en salud, de los siguientes elementos estructurales, íntegramente o de modo predominante: (i) universidad profesional; (ii) estructurada en facultades; (iii) formación intervencionista; (iv) orientada a la disciplina; (v) régimen de progresión lineal; (vi) pedagogía analítica; (vii) segregada por profesiones; (viii) tendiente a la especialización.

Como programa sistemático de investigación, sería pertinente y oportuno verificar, de hecho, su vigencia en la mayoría de las universidades, en otros países de América Latina. Sin duda, estudios de casos de institucionalización de la educación superior y de la formación médica, directa o indirectamente influenciados por la cultura francesa, produjeron informaciones y conocimiento, con un reducido grado de sistematización, a la espera de la compilación metódica, organización sistemática y análisis pertinente. No obstante, para una apreciación de esa dimensión sociopolítico-institucional, se recomienda la conducción de estudios comparativos de estructura organizacional y de marco regulatorio de las instituciones de enseñanza superior, con el objetivo de producir tipologías capaces de establecer los diferentes formatos asumidos por la reforma Cabanis en distintos contextos culturales, geopolíticos e históricos.

Finalmente, podemos considerar también plausible y robusta la hipótesis de que, además de sus raíces cabanistas latentes y denegadas, la universidad latinoamericana (incluyendo, de forma prioritaria, a la universidad argentina), después de todos estos años, permanece atada a la restauración bonapartista. De confirmarse esta situación, el contexto latinoamericano revelaría un aspecto intrigante, quizá un enigma histórico, pues reafirma el mantenimiento de diferentes variantes de un modelo de enseñanza superior (geopolíticamente referenciado -francés liberal burgués- e ideológicamente datado, precapitalista) que, en gran medida, fue abandonado en su matriz europea. Una comprensión, incluso limitada y parcial, de ese supuesto anacronismo puede revelar contradicciones de la universidad latinoamericana, en articulación orgánica con procesos políticos e institucionales de reproducción de las desigualdades económicas e inequidades sociales, solo posibles con la complicidad y activa acción de las instituciones de educación superior actuantes en esos países.

\section{AGRADECIMIENTOS}

Este artículo es parte de una investigación mayor titulada "Inovações curriculares e modelos pedagógicos como indutores de Competência Tecnológica Crítica (CTC) e Qualidade no Cuidado em Saúde (QCS) na Educação Médica", financiada por Institutos Nacionais de Ciência e Tecnologia, Ministério da Ciência, Tecnologia, Inovações e Comunicações, Conselho Nacional de Desenvolvimento Científico e Tecnológico, Coordenação de Aperfeiçoamento de Pessoal de Nivel Superior) (INCT-MCTI/CNPq/CAPES) No. 16/2014.

A la Coordenação de Aperfeiçoamento de Pessoal de Nivel Superior, por la beca de productividad científica (nivel I-A) otorgada a Naomar Almeida Filho, proceso 304671/2016-9.

\section{REFERENCIAS BIBLIOGRÁFICAS}

1. Charle C, Verger J. História das universidades. São Paulo: Editora UNESP; 1996.

2. Backhaus J. The university according to Humboldt and the alternatives. En: Backhaus J, (ed.). The university according to Humboldt: history, policy, and future possibilities. Berlin: Springer; 2015. p. 5-10.

3. Weinberg G. Modelos educativos em el desarrollo histórico de América Latina. Santiago: CEPAL, UNESCO, PNUD; 1981.

4. Almeida-Filho N. Impacto da reforma Cabanis no ensino médico do Brasil: ensaio de arqueologia neofoucaultiana. História, Ciências, Saúde-Manguinhos. (En edición 2018). 
5. Verneuil Y. L'école et la laïcité, de I'Ancien Régime à nos jours: enjeux du passé, enjeux dépassés? Tréma. 2012;37:130-143.

6. Weisz G. The emergence of modern universities in France, 1863-1914. Princeton: Princeton University Press; 1983.

7. Barnard H. Education and the French Revolution. Cambridge: Cambridge University Press; 1969.

8. Condorcet NC. Rapport et projet de décret sur I'organisation générale de l'instruction publique: présenté à l'Assemblée Nationale, au nom du Comité d'Instruction publique [Internet]. Paris: De L'imprimerie Nationale; 1792 [citado 1 nov 2018]. Disponible en: https://tinyurl.com/yd6rh7qy.

9. Williams E. The physical and the moral: anthropology, physiology, and philosophical medicine in France, 1750-1850. Cambridge: Cambridge University Press; 1994.

10. Pouliquen Y. Cabanis, un idéologue de Mirabeau à Bonaparte. Paris: Odile Jacob; 2013.

11. Destutt de Tracy ALC. Eléments d'idéologie [Internet]. Paris: A.F. Stella; 1803 [citado 1 nov 2018]. Disponible en: https://tinyurl.com/y8w8bhvm.

12. Cabanis PJG. Rapports du physique et du moral de l'homme [Internet]. Paris: Crapart, Caille et Ravier; 1802 [citado 1 nov 2018]. Disponible en: https://tinyurl.com/y7ypneut.

13. Cabanis PJG. Coup d'oeil sur les Revolutions et Réforme de la Médecine [Internet]. Paris: Firmin Didot; 1823 [citado 1 nov 2018]. Disponible en: https://tinyurl.com/y85ch5tl.

14. Saad M. Cabanis, comprendre I'homme pour changer le monde. Paris: Classiques Garnier ; 2016.

15. Staum M. Cabanis: enlightenment and medical philosophy in the French Revolution. Princeton: Princeton University Press; 1980.

16. Ramsey M. Professional and popular medicine in France, 1770-1848. Cambridge: Cambridge University Press; 1988.

17. Brockliss L, Jones C. The medical world of Early Modern France. Oxford: Clarendon Press; 1997.

18. Foucault M. O Nascimento da Clínica. Rio de Janeiro: Forense Universitária; 2011.

19. Schmitt S. From physiology to classification: comparative anatomy and Vicq d'Azyr's plan of reform for life sciences and medicine (1774-1794). Science in Context. 2009;22(2):145-193.
20. Crosland M. The Officiers de Santé of the French Revolution: a case study in the changing language of medicine. Medical History. 2004;48 (2):229-244.

21. Rosen G. The Philosophy of ideology and the emergence of modern medicine in France. Bulletin of the History of Medicine. 1946;20:328-339.

22. Rey R. L'Ecole de santé de Paris sous la Révolution: transformation et innovation. Histoire de l'Education. 1993;57:23-57.

23. Tabuteau D. Pouvoirs publics et professions de santé. Les Tribunes de la Santé. 2010;1(26):103121. doi: 10.3917/seve.026.0103.

24. Cabanis PJG. Rapport fait au nom de la Commission d'instruction publique, et projet de résolution, sur un mode provisoire de police médicale [Internet]. Paris: Represéntants du Peuple; 1798 [citado 1 nov 2018]. Disponible en: https://tinyurl. com/y77wkrjh.

25. Almeida-Filho N. Reforma Cabanis (Circa 1800): uma reforma universitária sem universidades, esquecida na história da educação. Revista Lusófona de Educação. 2018;39(39):41-54.

26. Habinek J. State-Building and the Origins of Disciplinary Specialization in Nineteenth-Century Germany [Internet]. En: American Sociological Association Annual Conference; 2010 [citado 1 nov 2018]; Atlanta. Disponible en: https://tinyurl.com/ y7cevldv.

27. Weisz G. Divide and conquer: a comparative history of medical specialization. Oxford: Oxford University Press; 2006.

28. Prost A. Regards historiques sur l'éducation en France, XIXe-XXe siècles. Paris: Belin; 2007.

29. Cabanis PJG. Compendio histórico de las revoluciones y reforma de la medicina. Madrid: Imprenta de Repullé; 1820.

30. Buchbinder P. Historia de las universidades argentinas. Buenos Aires: Sudamericana; 2010.

31. Halperin Donghi T. Historia de la Universidad de Buenos Aires. Buenos Aires: Eudeba; 2013.

32. Myers J. Las paradojas de la opinión: El discurso político rivadaviano y sus dos polos: el "gobierno de las luces" y la opinión pública, reina del mundo. En: Sabato H, Lettieri A, (comp.). La vida política en la Argentina del siglo XIX: Armas, votos y voces. Buenos Aires: Fondo de Cultura Económica; 2003. p. 75- 95. 
33. Ternavasio M. Las reformas rivadavianas en Buenos Aires y el Congreso General Constituyente. En: Goldman N, (dir.). Nueva Historia Argentina: Revolución, República, Confederación (1806-1852). T. 3. Sudamericana: Buenos Aires; 1998. p.161-197.

34. Piccirilli R. Rivadavia y su tiempo. Vol. 1-2. Buenos Aires: Ediciones Peuser; 1943.

35. Di Pasquale M. Prensa, política y medicina en Buenos Aires: Un estudio de La Abeja Argentina, 1822-1823. Estudios de Teoría Literaria-Revista digital: artes, letras y humanidades. 2016;5(9):119-136.

36. Di Pasquale M. La recepción de la Idéologie y su impacto en la educación médica porteña, 1821-1840. Revista de Historia de la Medicina y Epistemología Médica. 2012;IV(1):1-20.

37. Fernández de Agüero JM. Principios de ideología: Primer Curso de filosofía dictado en la Universidad de Buenos Aires (1822-1827). T. 1. Buenos Aires: Universidad de Buenos Aires, Facultad de Filosofía y Letras; 1940.

38. Di Pasquale M. La recepción de la Idéologie en la Universidad de Buenos Aires: El caso de Juan Manuel Fernández de Agüero. Prismas, Revista de Historia Intelectual. 2011;(15):63-86.

39. Ternavasio M. La revolución del voto: Política y elecciones en Buenos Aires, 1810-1852. Buenos Aires: Siglo XXI Editores; 2002.

40. Di Meglio G. ¡Viva el bajo pueblo! La plebe urbana de Buenos Aires y la política entre la Revolución de Mayo y el rosismo. Buenos Aires: Prometeo; 2006.
41. Myers J. Orden y virtud. El discurso republicano en el régimen rosista. Buenos Aires: Universidad Nacional de Quilmes; 1995.

42. Di Pasquale M. Diego Alcorta y la difusión de saberes médicos en Buenos Aires, 1821-1842. Dynamis, Acta Hispanica ad Medicinae Scientiarumque Historiam Illustrandam. 2014;34(1):125-146.

43. Alcorta D. Disertación sobre la manía aguda. [Tesis de grado]. Buenos Aires: Universidad de Buenos Aires; 1827.

44. Alcorta D. Lecciones de filosofía. Buenos Aires: Fondo Nacional de las Artes; 2001.

45. Grmek M, Bernabeo R. La machine du corps. En: Grmek M, (dir.). Histoire de la pensé médicale en Occident: De la Renaissance aux Lumièrs. T. 2. Paris: Seuil;1997. p. 7-36.

46. Anónimo. Un inglés: cinco años en Buenos Aires 1820-1825. Buenos Aires: Hyspamérica; 1986.

47. Fielbaum A. La filosofía ante la crisis: Alejandro Korn y la reforma universitaria de las humanidades. Estudios de Filosofía. 2017;56:26-48.

48. Biagini H. La reforma universitaria: Antecedentes y consecuentes. Buenos Aires: Leviatán; 2000.

49. Almeida-Filho N. Reconhecer Flexner: inquérito sobre produção de mitos na educação médica no Brasil contemporâneo. Cadernos de Saúde Pública. 2010;26(12):2234-2249. doi: 10.1590/S0102-311X2010001200003.

\section{FORMA DE CITAR}

Almeida-Filho N, Di Pasquale M. El impacto de la reforma Cabanis en la formación histórica de la universidad argentina y en la educación superior en salud. Salud Colectiva. 2019;15:e2106. doi: 10.18294/sc.2019.2106.

Recibido: 26 de noviembre de 2018 | Aprobado: 27 de diciembre de 2018

Esta obra está bajo una licencia de Creative Commons Reconocimiento-NoComercial 4.0 Internacional. Reconocimiento - Permite copiar, distribuir y comunicar públicamente la obra. A cambio, se debe reconocer y citar al autor original. No Comercial - Esta obra no puede ser utilizada con finalidades comerciales, a menos que se obtenga el permiso. 